\title{
Apexificación de un molar permanente a través de silicato tricálcico
} Apexification of a permanent molar with tricalcium silicate

https://doi.org/10.37135/ee.04.08.05

\begin{abstract}
Autoras:
María José Massón Palacios ${ }^{1,2}$ - (https://orcid.org/0000-0001-7690-4733)

Miriam Alejandra Aguilar Ortiz ${ }^{3}$ - (https://orcid.org/0000-0001-5278-7755)

Juan Marcos Parise Vasco ${ }^{1,2}$ - (https://orcid.org/0000-0002-5223-3370)

Ana del Carmen Armas Vega ${ }^{2}$ - (https://orcid.org/0000-0003-3800-8166)
\end{abstract}

${ }^{1}$ Centro de Investigación de Salud Pública y Epidemiología Clínica. Quito-Ecuador.

${ }^{2}$ Universidad UTE Quito-Ecuador.

${ }^{3}$ Clínica Odontológica Masterdent. Quito - Ecuador.

Autor de correspondencia: María José Massón Palacios. Universidad Tecnológica Equinoccial, Rumipamba y Bourgeois, Quito, Ecuador, código postal: 170147. Email: maria.masson@ute.edu.ec.

\section{RESUMEN}

Paciente pediátrico de 9 años y género masculino, que acudió para valoración odontológica por presentar dolor dental tras ingerir alimentos fríos. El diagnóstico definitivo quedó establecido como pulpitis reversible del molar 3.6, decidiendo conducta terapéutica de recubrimiento pulpar directo con silicato tricálcico para lograr apexificación. La decisión clínica resultó exitosa, el seguimiento de la evolución permitió apreciar que el diente mantuvo su vitalidad, las raíces continuaron desarrollándose y no aparecieron signos o síntomas de lesiones periapicales.

Palabras clave: apexificación, pulpotomía, silicato tricálcico.

\section{ABSTRACT}

9-year-old pediatric male patient, who came for dental evaluation because of dental pain after eating cold food. The definitive diagnosis was established as reversible pulpitis of the molar 3.6, deciding on the therapeutic behavior of the direct pulp coating with tricalcium silica to achieve apexification. The clinical decision was successful, the follow-up of the evolution identified the affected tooth as vital, the roots continued to develop, and no signs or symptoms of periapical lesions appeared.

Keywords: apexification, pulpotomy, tricalcium silicate. 


\section{INTRODUCCIÓN}

A nivel mundial, la prevalecía de las alteraciones pulpares en dientes permanentes jóvenes supera el $34,1 \%{ }^{(1)}$ producto de la disbiosis dental asociada a dieta, microrganismos y tiempo ${ }^{(2)}$ o durante los procedimientos restauradores que desencadenan la exposición de la pulpa dental con síntomas dolorosos, compatibles con aquellos de la pulpitis irreversible. ${ }^{(3)}$ Cuando esta patología se presenta en un diente con ápice inmaduro o en amplios y abiertos, puede paralizar la formación de dentina, el crecimiento radicular ${ }^{(4)} \mathrm{e}$ incluso generar necrosis pulpar, convirtiéndose en un desafío clínico. ${ }^{(5)}$

Ante una exposición pulpar en un diente permanente joven, se requiere de un tratamiento para la regeneración de ese tejido y el cierre apical, el ensanchamiento del conducto durante su preparación constituye un proceso fundamental y muy delicado por el elevado riesgo de factura. ${ }^{(6)}$

Al respecto, la pulpotomía resulta un tratamiento recomendado, su objetivo es eliminar toda la pulpa coronal y recubrir desde la que se encuentra adyacente hasta la que se ubica en la raíz, preservándola dentro del conducto radicular y las raíces de los dientes inmaduros para que pueden continuar creciendo; a ese proceso se le define como apexificación. ${ }^{(7)}$ En este es necesario utilizar el material biocompatible con propiedades antimicrobianas y que favorezca la cicatrización y regeneración del complejo dentinopulpar, ${ }^{(8,9)}$ mediante la inducción y diferenciación de nuevos odontoblastos, ${ }^{(10,11)}$ bloqueando la contaminación por microorganismos orales; además de mantener la vitalidad y función pulpar. ${ }^{(11)}$

La tendencia actual está dirigida al uso de materiales con propiedades bioactivas que emulen las funciones de los tejidos biológicos, entre los que se encuentra el cemento restaurador a base de silicato tricálcio (Biodentine Septodont), el que está compuesto por carbonato de calcio y óxido de circonio y debe ser mezclado mediante una sustancia líquida integrada por agua, cloruro de calcio para acelerar el fraguado y policarboxilato modificado como agente plastificante. Sus propiedades mecánicas son similares a la dentina. ${ }^{(12,13)}$

La utilización del cemento silicato de calcio, ayuda a mantener el tejido vital de la pulpa, generando el desarrollo, la propagación y diferenciación de las células madre para su regeneración, estimulando la producción de dentina reparadora. Puede utilizarse en coronas y raíces; en el caso de la primera, protege la pulpa, cierra temporalmente, favorece el manejo de caries profundas, sirve como relleno cervical y puede usarse durante pulpotomías. ${ }^{(12)}$

El presente artículo tiene como propósito reportar un caso de pulpotomía, debido a una exposición pulpar producida por caries profunda en un molar permanente joven, utilizando silicato tricálcico para lograr apexificación. 


\section{PRESENTACION DEL CASO}

Paciente pediátrico de 9 años de edad y género masculino, que acude para valoración odontológica acompañado de su madre, por presentar dolor dental tras ingerir alimentos fríos; durante la anamnesis, se refirió la ausencia de antecedentes patológicos personales o familiares de interés.

Al examen extraoral, presentó tejidos faciales dentro de los parámetros de normalidad; mientras que el intraoral permitió apreciar una lesión cariosa profunda oclusal en el primer molar izquierdo inferior.

El examen radiográfico refleja una sombra radiolúcida a nivel coronal compatible con impresión diagnóstica de caries oclusal severa, ápices radiculares en estado de nolla 8 (figura 1).

Figura 1. Radiografía inicial estado de nolla 8 unidad dental 3.6

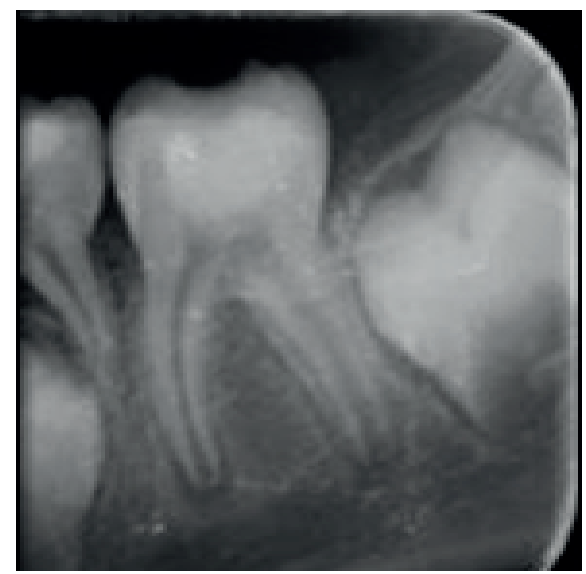

Fuente: historia clínica del paciente

Las pruebas de vitalidad pulpar mostraron dolor transitorio, el que se incrementa a la percusión a nivel del diente 3.6. Las respuestas de sensibilidad resultan positivas, rápidas e intensas ante estímulo de frío y negativas al calor.

El diagnóstico definitivo quedó establecido como pulpitis reversible del molar 3.6. Así, se trazaron las alternativas de tratamiento y fueron discutidas con la madre del niño; en conjunto, se decidió realizar recubrimiento pulpar directo con silicato tricálcico para lograr apexificación. El paciente y su tutora legal también fueron instruidos sobre buenas prácticas de higiene bucal y manejo dietético. 
Tras la firma del respectivo consentimiento informado, se procedió a la ejecución del tratamiento:

Primera cita, acondicionamiento del medio bucal mediante profilaxis.

Segunda cita (figura 2), bajo régimen de aislamiento absoluto y administración de anestesia infiltrativa, se retiró la restauración provisional y el tejido afectado por la caries. La remoción en la zona más profunda se realizó con una pieza de alta velocidad y fresa diamantada redonda mediana, luego se usó una cuchareta para retirar la dentina afectada. Durante ese proceso se produjo una exposición pulpar, que requirió de un lavado abundante utilizando solución fisiológica y el secado de las superficies con torundas estériles.

Posteriormente, la pulpa cameral fue removida en su totalidad y se colocó cemento restaurador a base de silicato tricálcico (Biodentine Septodont) en la entrada de los conductos radiculares, con el fin de mantener la vitalidad de la pulpa e inducir la apexificación. El medicamento fue administrado en la entrada de los conductos radiculares siguiendo las instrucciones del fabricante. Luego de 5 minutos de fraguado, se colocó una capa de ionómero de vidrio de base (Vitremer $3 \mathrm{M}$ ). Finalmente, se restauró la cavidad utilizando resina nanohibrida color A1 (FILTEX 350XT $3 \mathrm{M})$.

Figura 2. Secuencia de tratamiento en la segunda cita

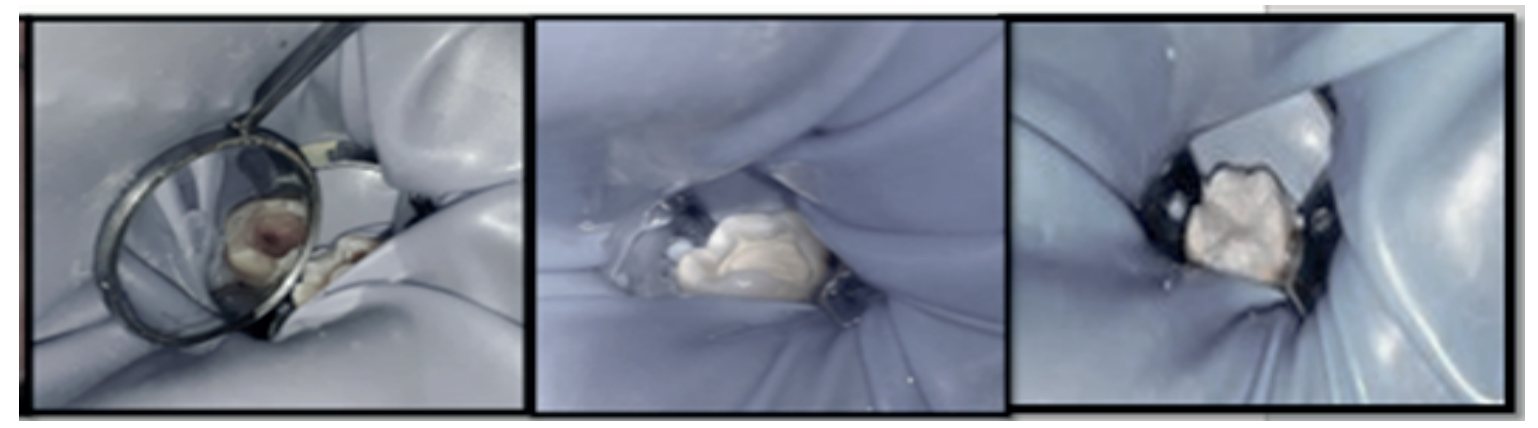

Fuente: historia clínica del paciente

La evolución del paciente se realizó mediante controles clínicos mensuales durante seis meses. Las pruebas de percusión y palpación posibilitaron determinar la ausencia de los signos o síntomas de la patología pulpar, así como de fistulas o inflamación. Las radiografías periapicales (figura 3), tomadas a intervalos de 30 días, mostraron resultados satisfactorios en relación con la formación radicular, el engrosamiento de las paredes radiculares y el cierre apical. 
Figura 3. Secuencias de Rx de control de la evolución ordenadas de izquierda a derecha: a los dos, tres y seis meses respectivamente

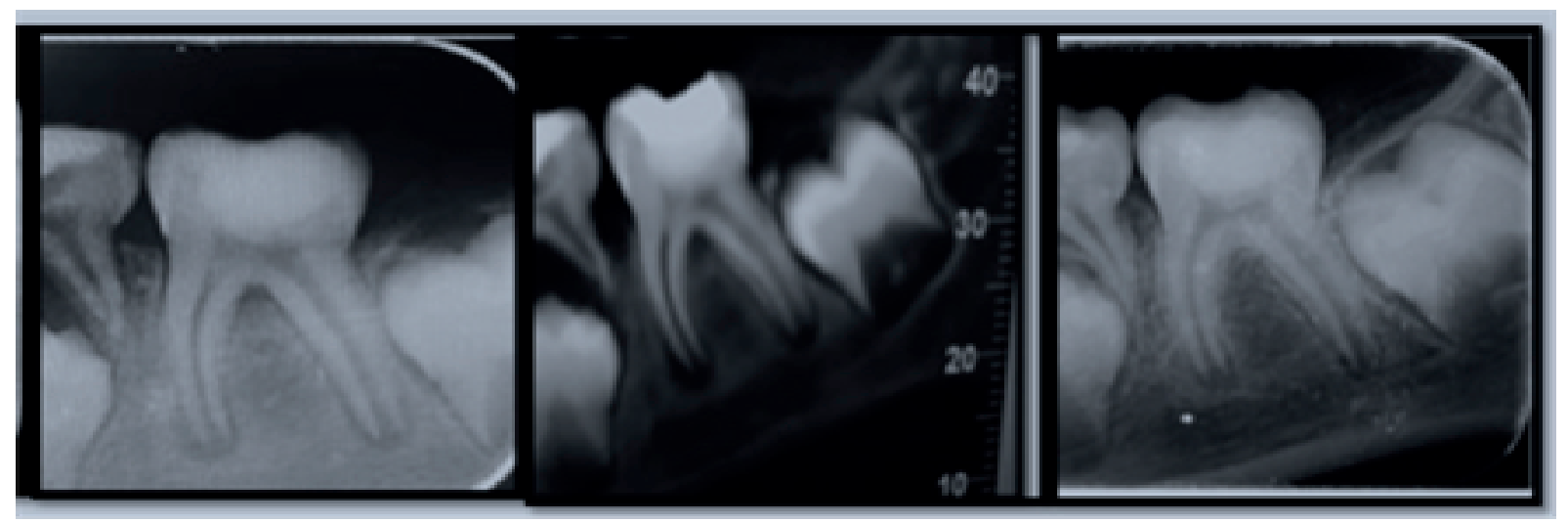

Fuente: historia clínica del paciente

\section{DISCUSIÓN}

El tratamiento realizado siguió el protocolo para pulpotomía aplicando un material bioactivo (Biodentine Septodont), para mantener la vitalidad de la pulpa radicular y garantizar la apexificación. Los resultados iniciales y su seguimiento durante 6 meses fueron exitosos, pero resulta recomendable mantener la valoración de la evolución con el propósito de detectar tempranamente la ausencia de sintomatología pulpar, que derive en irreversibilidad y procedimiento endodóntico. Los efectos logrados con silicato tricálcico resultaron comparables a los obtenidos en otro estudio sobre pulpotomía, donde se utilizó mineral trióxido agregado (MTA). ${ }^{(14)}$

La exposiciones pulpares debido a caries profundas son eventos frecuentes en niños y adolescentes, los dientes en proceso de maduración son vulnerables ante un cuidado inadecuado y pueden requerir un tratamiento complicado precozmente. ${ }^{(15)}$ Antes de la aparición de este tipo de biomateriales, esas patologías se consideraban irreversibles aunque no presentaran síntomas. ${ }^{(4)}$ El desarrollo de esos medicamentos con propiedades de biocompatibilidad y bioactividad que, al entrar en contacto directo con el tejido pulpar, inducen el desarrollo de dentina reparativa, contribuyendo al cierre apical y a la aparición de tratamientos menos invasivos en pulpas vitales dañadas. ${ }^{(16)}$

La terapia de recubrimiento pulpar persigue el objetivo de mantener todas las funciones de esa estructura orgánica del diente, especialmente el potencial regenerativo. Aunque existen diversos materiales similares, la decisión en cuanto a la selección del silicato tricálcico resultó acertada debido a las ventajas clínicas; ${ }^{(17)}$ entre las que destaca un menor tiempo de fraguado en compara- 
ción con el MTA, lo que favorece su manejo y resistencia, ${ }^{(18)}$ además de reducir el riesgo de pérdida parcial de material y alteración de la interfaz durante la fase de finalización del procedimiento. ${ }^{(19,20)}$

En varias pruebas, el silicato tricálcico exhibió una menor porosidad que el MTA. ${ }^{(21)}$ según Luo et al., ${ }^{(22)}$ quien usó el mismo material empleado en el presente caso en dientes permanentes jóvenes, este produce un incremento significativo en la proliferación, migración y adhesión de las células madre de la pulpa dental humana, cuando se aplica directamente, además de promover la mineralización generando un puente de dentina reaccionaria densa al ponerse en contacto con la pulpa.

Durante la pulpotomía, el facultativo valoró acertadamente el remanente de tejido pulpar en cuanto a la vitalidad de este; su capacidad de regeneración y reparación puede verse afectada por la acción de las bacterias durante el periodo de recuperación, que requerirá de elevadas defensas en el diente tratado. ${ }^{(11)}$

La edad del paciente en cuestión representó una característica favorable para su evolución. En la medida que los individuos se hacen mayores, la pulpa dental se hace más fibrosa y menos regenerativa y vital ante las lesiones; mientras que, en los dientes permanentes jóvenes existe un mejor suministro de sangre y el mecanismo de defensa es más eficiente en la resistencia contra infecciones bacterianas. ${ }^{(23)}$

\section{CONCLUSIÓN}

El uso de silicato tricálcico arrojó buenos resultados en la apexicificación; el seguimiento de la evolución del paciente permitió apreciar que el diente mantuvo su vitalidad, las raíces continuaron desarrollándose y no se aparecieron signos o síntomas de lesiones periapicales.

Conflictos de interés: los autores declaran que no existen.

\section{Declaración de contribución:}

María José Massón Palacios y Miriam Alejandra Aguilar Ortiz escogieron el caso y recolectaron la información; mientras que Juan Marcos Parise Vasco y Ana del Carmen Armas Vega participaron en la discusión y análisis del mismo; además, que de conjunto redactaron el artículo. 


\section{REFERENCIAS BIBLIOGRÁFICAS}

1. Kassebaum NJ, Smith AGC, Bernabé E, Fleming TD, Reynolds AE, Vos T, et al. Global, Regional, and National Prevalence, Incidence, and Disability-Adjusted Life Years for Oral Conditions or 195 Countries, 1990-2015: A Systematic Analysis for the Global Burden of Diseases, Injuries, and Risk Factors. J Dent Res. 2017; 96(4): 380-387.

2. Fontana M, Young DA, Wolff MS, Pitts NB, Longbottom C. Defining dental caries for 2010 and beyond. Vol. 54, Dental clinics of North America. United States; 2010. p. $423-40$.

3. Gulabivala K, Ng YL. Endodontics E-Book. Barcelona: Elsevier Health Sciences; 2014.

4. Stanley HR. Pulp capping: conserving the dental pulp--can it be done? Is it worth it? Oral Surg Oral Med Oral Pathol. 1989 Nov; 68(5): 628-639.

5. Sahli C, Aguadé EB. Endodoncia Técnicas clínicas y bases científicas. 3ra ed. Barcelona: Masson; 2014.

6. Laslami K, Dhoum S, Karami M, Jabri M. EC DENTAL SCIENCE Case Report Direct Pulp Capping with Bioactive Material: Biodentine. Ec Dent Sci. 2017; 2: 75-83.

7. DeRosa TA. A retrospective evaluation of pulpotomy as an alternative to extraction. Gen Dent. 2006; 54(1): 37-40.

8. Zhang W, Yelick PC. Vital Pulp Therapy - Current Progress of Dental Pulp Regeneration and Revascularization. Int J Dent. 2010; 2010: 1-9.

9. Ranly DM, Garcia-Godoy F. Current and potential pulp therapies for primary and young permanent teeth. J Dent. 2000 Mar; 28(3): 153-161.

10. Fuks AB. Vital Pulp Therapy with New Materials for Primary Teeth: New Directions and Treatment Perspectives. J Endod. 2008; 34(7 SUPPL.).

11. Tziafas D, Belibasakis G, Veis A, Papadimitriou S. Dentin regeneration in vital pulp therapy: design principles. Adv Dent Res. 2001 Aug; 15: 96-100.

12. Muhamad A-H, Abdulgani A, Abdulgani M, Georges C, Ayah J. Management of Open Apex in Permanent Teeth with Biodentine. IOSR J Dent Med Sci. 2017; 16(2): 135-140. 
13. Hincapié-Narváez S, Valerio-Rodriguez AL. Biodentine: Un nuevo material en terapia pulpar/ Biodentine: A New Material for Pulp Therapy. Univ Odontol. 2015; 34(73): 69-76.

14. Qudeimat MA, Alyahya A, Hasan AA. Mineral trioxide aggregate pulpotomy for permanent molars with clinical signs indicative of irreversible pulpitis: a preliminary study. Int Endod J. 2017 Feb; 50(2): 126-134.

15. Ridell $\mathrm{K}$, Olsson $\mathrm{H}$, Mejàre I. Unrestored dentin caries and deep dentin restorations in Swedish adolescents. Caries Res. 2008; 42(3): 164-170.

16. Solanki NP, Venkappa KK, Shah NC. Biocompatibility and sealing ability of mineral trioxide aggregate and biodentine as root-end filling material: A systematic review. J Conserv Dent. 2018; 21(1): 10-15.

17. Malkondu Ö, L MKK, L EK. A Review on Biodentine, a Contemporary Dentine Replacement and Repair Material. 2014; 2014.

18. Shafaee H, Alirezaie M, Rangrazi A, Bardideh E. Comparison of the success rate of a bioactive dentin substitute with those of other root restoration materials in pulpotomy of primary teeth: Systematic review and meta-analysis. J Am Dent Assoc. 2019 Aug; 150(8): 676-688.

19. Kaur M, Singh H, Dhillon JS, Batra M, Saini M. MTA versus Biodentine : Review of Literature with a Comparative Analysis. 2017; 11(8): 3-7.

20. Grech L, Mallia B, Camilleri J. Investigation of the physical properties of tricalcium silicate cement-based root-end filling materials. Dent Mater [Internet]. 2012 [citado 13 Feb 2019]; 29(2): 20-28. Disponible en: http://dx.doi.org/10.1016/j.dental.2012.11.007.

21. Aydemir, S., Cimilli, H., Gerni, P.M., Bozkurt, A., Urucoglu, H., Chandler, N., \& Kartal, N. (2016). Comparison of the Sealing Ability of Biodentine, iRoot BP Plus and Mineral Trioxide Aggregate. Cumhuriyet Dental Journal [Internet]. 2016 [citado 13 Feb 2019]; 19(2): 166-171. Disponible en: Doi: 10.7126/cdj.58140.5000144905.

22. Luo Z, Kohli MR, Yu Q, Kim S, Qu T, He W. Biodentine induces human dental pulp stem cell differentiation through mitogen-activated protein kinase and calcium-/calmodulin-dependent protein kinase II pathways. J Endod. 2014 Jul; 40(7): 937-942. 
23. Claus I, Laureys W, Cornelissen R, Dermaut LR. Histologic analysis of pulpal revascularization of autotransplanted immature teeth after removal of the original pulp tissue. Am J Orthod Dentofacial Orthop. 2004 Jan; 125(1): 93-99.

Recibido: 3 de enero de 2020

Aprobado: 16 de mayo de 2020 